\title{
Unscheduled Emergency Visits after Cardiac Devices Implantation: Comparison between Cardioverter Defibrillators and Cardiac Resynchronization Therapy Devices in less than one year of Follow Up
}

\author{
Stefan Warpechowski Neto, ${ }^{1}$ Laura Lessa Gaudie Ley, ${ }^{2}$ Eduardo Dytz Almeida, ${ }^{1}$ Marco Aurélio Lumertz Saffi, ${ }^{3}$ \\ Luiza Zwan Dutra, ${ }^{1}$ Antonio Lessa Gaudie Ley, ${ }^{1}$ Roberto Tofani Sant `Anna, ${ }^{1}$ Gustavo Glotz de Lima, ${ }^{1}$ \\ Renato Abdala Karam Kalil, ${ }^{1}$ Tiago Luiz Luz Leiria' ${ }^{10}$ \\ Instituto de Cardiologia / Fundação Universitária de Cardiologia (IC/FUC), ${ }^{1}$ Porto Alegre, RS - Brazil \\ Pontifícia Universidade Católica do Rio Grande do Sul, ${ }^{2}$ Porto Alegre, RS - Brazil \\ Hospital de Clínicas de Porto Alegre, ${ }^{3}$ Porto Alegre, RS - Brazil
}

\begin{abstract}
Background: The use of Cardiovascular Implantable Electronic Devices (CIED), such as the Implantable Cardioverter Defibrillator (ICD) and Cardiac Resynchronization Therapy (CRT), is increasing. The number of leads may vary according to the device. Lead placement in the left ventricle increases surgical time and may be associated with greater morbidity after hospital discharge, an event that is often confused with the underlying disease severity.
\end{abstract}

Objective: To evaluate the rate of unscheduled emergency hospitalizations and death after implantable device surgery stratified by the type of device.

Methods: Prospective cohort study of 199 patients submitted to cardiac device implantation. The groups were stratified according to the type of device: ICD group $(n=124)$ and CRT group $(n=75)$. Probability estimates were analyzed by the Kaplan-Meier method according to the outcome. A value of $p<0.05$ was considered significant in the statistical analyses.

Results: Most of the sample comprised male patients $(71.9 \%)$, with a mean age of $61.1 \pm 14.2$. Left ventricular ejection fraction was similar between the groups (CRT $37.4 \pm 18.1$ vs. ICD $39.1 \pm 17.0, p=0.532$ ). The rate of unscheduled visits to the emergency unit related to the device was $4.8 \%$ in the ICD group and $10.6 \%$ in the CRT group (p=0.20). The probability of device-related survival of the variable "death" was different between the groups $(p=0.008)$.

Conclusions: Patients after CRT implantation show a higher probability of mortality after surgery at a follow-up of less than 1 year. The rate of unscheduled hospital visits, related or not to the implant, does not differ between the groups. (Arq Bras Cardiol. 2019; 112(5):491-498)

Keywords: Defibrillators, Implantable; Cardiac-Gated Imaging Techniques; Cardiac Resynchronization Therapy Devices; Patient Readmission; Mortality.

\section{Introduction}

In the cardiovascular disease scenario, patients with reduced left ventricular ejection fraction (LVEF) after acute myocardial infarction show an increased risk of sudden death related to cardiac arrhythmia. The use of Cardiovascular Implantable Electronic Devices (CIED), such as Implantable Cardioverter Defibrillators (ICDs), has shown to be beneficial in improving survival rates in this patient profile. ${ }^{1}$ The Cardiac Resynchronization Therapy (CRT) also demonstrates benefits in reducing hospitalization rates, improving ventricular function, as well as decreasing mortality in the context of heart failure (HF). ${ }^{2,3}$

Mailing Address: Tiago Luiz Luz Leiria •

Av. Princesa Isabel, 370. Postal Code 90620-000, Santana. Porto Alegre, RS - Brazil

E-mail: pesquisa.leiria@gmail.com, editoracao-pc@cardiologia.org.br Manuscript received June 04, 2018, revised manuscript September 05 2018, accepted September 05, 2018

DOI: 10.5935/abc.20190018
During the CIED implantation, according to the clinical indication, it is necessary to use one, two or even three intracardiac leads. In CRT, the difficulty in cannulating the coronary sinus, or the lack of an adequate venous branch for this purpose, tends to increase the surgical procedure complexity, which may be associated with greater morbidity in the follow-up after hospital discharge - a situation often attributed or confused with the underlying disease severity. Regardless of the implantation route used for left ventricular estimation, we know there are lead dislodgement and dysfunction rates of approximately $5 \%{ }^{4}$ after the surgery, and the presence of a higher number of leads makes the probability of this type of event occurrence even higher. On the other hand, unscheduled emergency visits related to CIED occur, not necessarily because of the leads, in up to $12 \%$ of patients undergoing this type of therapy. ${ }^{4,5}$ Local data that assess the rate of unscheduled hospital visits related to the implants are limited to the southeast region, and there is no recent literature disclosing data from the southern region of the country. The present study aims to contribute to this issue. 


\section{Methods}

\section{Study design}

This was a prospective single-center cohort study developed in a high-complexity cardiology hospital located in southern Brazil.

\section{Population}

Patients aged 18 years or older submitted to ICD implantation or CRT from February 2014 to July 2015 were consecutively included. The cases of changes in generators without lead implantation were excluded.

\section{Data collection}

Data collection was performed from the moment immediately prior to the device implantation and, subsequently, at the time of visits to the emergency unit for medical care. The obtained data, recorded in the electronic medical record, were exported to an Excel database. The analyzed variables were clinical ones and related to the implant. The clinical variables were: (1) LVEF at the two-dimensional echocardiogram (Teicholtz or Simpson method, when indicated); (2) HF etiology, defined as valvular, ischemic or non-ischemic. In case of more than one etiology, we selected the one considered to be predominantly accountable for the condition; (3) patient functional class, classified according to the New York Heart Association classification. The variables related to the CIED implant were: (1) operative wound infections; (2) operative wound pain; (3) need to replace the leads; (4) upper limb venous thrombosis; and (5) device pocket hematoma.

\section{Outcomes}

The primary outcome was the occurrence of an unscheduled hospital visit related to the CIED implant. Visits resulting from HF worsening or progression were also considered for this analysis. The secondary outcome was all-cause mortality.

\section{Ethical Considerations}

This study was approved by the Research Ethics Committee of Instituto de Cardiologia - Fundação Universitária de Cardiologia do Rio Grande do Sul, under number 4983/14.

\section{Data analysis}

The Statistical Package for Social Sciences (SPSS), version 18.0, was used for the analyses based on the data stored in the Microsoft Excel spreadsheets. A two-tailed $p$ value of less than 0.05 was considered significant in the statistical analyses. Continuous variables with parametric distribution were expressed as mean \pm standard deviation, while nonparametric variables were shown as median and interquartile range. The comparisons were made with Student's t-test for independent samples in variables with central tendency distribution and with Mann-Whitney test in those considered to be asymmetric. Categorical variables were expressed as absolute (n) and relative (\%) frequencies and compared with the chi-square test. Probability estimates were calculated by the Kaplan-Meier method using long-rank. It was not possible to perform the multivariate analysis using Cox Regression due to the absence of events occurring in the ICD group, which prevented the calculation of the hazard ratio (HR).

\section{Results}

During the study period, 1,174 surgeries were performed for device implantation. Of these, 224 were for ICD/CRT, 25 of which were exclusively for generator change, and were excluded from the evaluation. Figure 1 shows patient inclusion flowchart. The final analysis was performed with 199 patients. Table 1 shows the characteristics of the assessed population. There was a higher prevalence of male individuals in both groups. The mean age was similar, as well as the ejection fraction. The non-ischemic cardiomyopathy was the most prevalent etiology in both groups. Most implants were performed through the Brazilian Unified Public Health System (SUS). There was a statistically significant difference between the functional classes, with a higher percentage of patients in class III in both groups. Of all procedures, 57\% were carried out for primary prevention of sudden death.

\section{Outcomes}

Regarding the outcomes, the rate of unscheduled visits to the emergency unit related to the device was $4.8 \%$ in the ICD group and $10.6 \%$ in the CRT group ( $p=0.20$ ). Operative wound pain was the most prevalent complication related to the device (Table 2 ).

Figure 2 shows the incidence of the primary outcome of emergency visits-free survival during a median follow-up of 285 days ( $p=0.214)$. The incidence of unscheduled visits related to clinical conditions (not related to device implantation) did not differ between the groups, being $28.2 \%$ in the ICD group and $18.6 \%$ in the CRT group $(p=0.17)$, including readmissions due to HF, as shown in Table 3.

Figure 3 shows the survival curve in both groups. There were 4 deaths in the CRT group and none in the ICD group. None of the deaths were related to the procedure itself. The causes of death were: 1 hemorrhagic stroke, 1 sudden death at home, 1 death due to multiple organ failure as a complication of infective endocarditis (secondary to a dental abscess, diagnosed 194 days after the implant) and 1 due to refractory HF.

\section{Discussion}

Artificial cardiac stimulation has shown significant benefits since its initial implantation in 1958, crossing generations in continuous technical evolution and extending its range from the atrioventricular conduction disorders to dyssynchrony reduction. However, it still shows a significant percentage of complications, despite almost sixty years of use. Currently, the volume of procedures for CIED implantation shows increasing annual rates, due to the technological evolution of the method, the increase in the indications and the higher number of eligible patients. ${ }^{6}$ At the same time, the greater longevity of the affected populations is a non-modifiable risk factor for long-term complications. Such a change in the scenario limits 


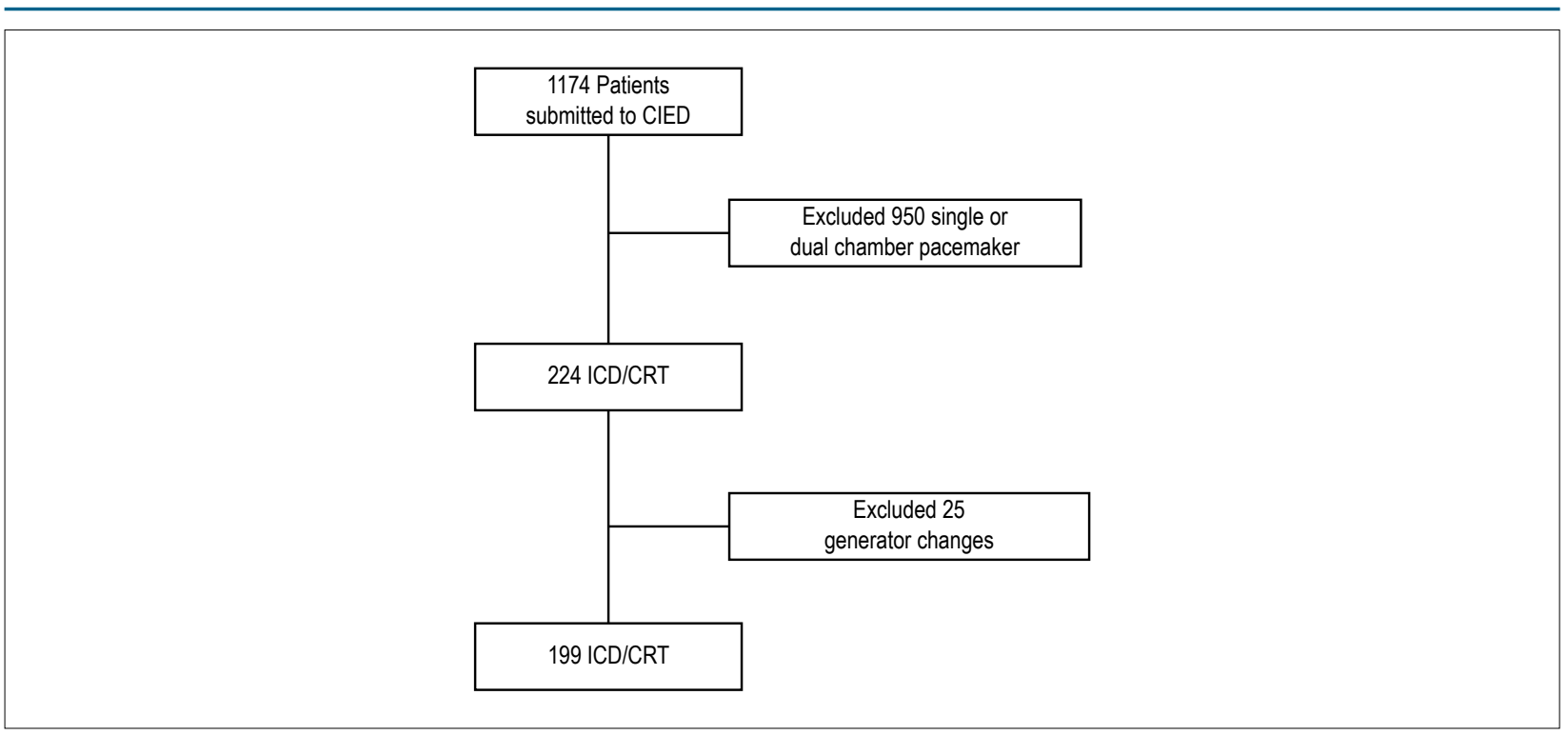

Figure 1 - Inclusion flowchart of the study patients. CIED: cardiac implantable electronic device. ICD: implantable cardioverter-defibrillator. CRT: cardiac resynchronization therapy.

Table 1 - Sample characteristics. Porto Alegre, RS

\begin{tabular}{|c|c|c|c|c|}
\hline Variable & Total $n=199$ & $I C D n=124$ & CRT-P/D n = 75 & $\mathrm{p}$ value \\
\hline Age, years* & $61.1 \pm 14.2$ & $61.1 \pm 14.3$ & $61.0 \pm 14.2$ & 0.963 \\
\hline Male gender $\uparrow$ & $143(71.9)$ & $94(75.8)$ & $49(65.3)$ & 0.153 \\
\hline $\operatorname{LVEF}(\%)^{*}$ & $38.4 \pm 17.4$ & $39.1 \pm 17.0$ & $37.4 \pm 18.1$ & 0.532 \\
\hline Etiology & & & & 0.043 \\
\hline Non-ischemic cardiomyopathy & $116(58.3)$ & $66(53.2)$ & $50(66.7)$ & \\
\hline Ischemic cardiomyopathy & $79(39.7)$ & $56(45.2)$ & $23(30.7)$ & \\
\hline Valvular etiology & $4(2)$ & $2(1.6)$ & $2(2.7)$ & \\
\hline Type of health care & & & & 0.349 \\
\hline SUS & $134(67.3)$ & $87(70.2)$ & $47(62.7)$ & \\
\hline Supplementary health care & $65(32.7)$ & $37(29.8)$ & $28(37.3)$ & \\
\hline \multicolumn{5}{|l|}{ Functional Class } \\
\hline I & $39(19.5)$ & $32(25.8)$ & $7(9.3)$ & 0.007 \\
\hline II & $24(12)$ & $13(10.4)$ & $11(14.6)$ & \\
\hline III & $72(36.1)$ & $36(29)$ & $36(48)$ & \\
\hline IV & $31(15.5)$ & $21(16.9)$ & $10(13.3)$ & \\
\hline
\end{tabular}

the comparison of current data with the first era of stimulation, not only by the device evolution curve, the implant technique and population factor alterations, but also by the database of previous records - many of them comprising only complications demanding surgical intervention. Over the years, the variability of complication definitions has become more homogeneous, with a further description of conservative management adverse effects, with the inaccuracies in temporal definition of events having been overcome, now dichotomized as early or late within a time frame of 2 months. ${ }^{6-8}$ The current series, many limited to the review of the last 20 years, indicate the first sixty days as the period with the highest incidence of complications, with rates that fluctuate around $10 \%$, in their majority. ${ }^{9,10}$

This study brings current national data on morbidity and mortality after ICD/CRT implantation. Our hospital is a tertiary cardiology center that performs approximately 1,000 device implants per year. The total incidence of complications related to the devices was $7 \%$ in the studied period, similar to that of other studies on the subject. ${ }^{11}$ Our sample had an incidence of cable dislocation, infections and mortality of $0.5 \%, 2.5 \%$ and $2 \%$, respectively. 
Table 2 - Outcomes of the study population. Device-related unscheduled emergency visit

\begin{tabular}{|c|c|c|c|c|}
\hline Variable & Total $n=199$ & $I C D$ n = 124 & CRT-P/D n = 75 & $p$ value \\
\hline Device-related unscheduled emergency visit" & $14(7 \%)$ & $6(4.8 \%)$ & $8(10.6)$ & 0.20 \\
\hline Device-related complications ${ }^{*}$ & & & & 0.45 \\
\hline Surgical wound infection & $5(2.5 \%)$ & $2(1.6 \%)$ & $3(4 \%)$ & \\
\hline Surgical wound pain & $6(3 \%)$ & $2(1.6 \%)$ & $4(5.3 \%)$ & \\
\hline Lead change & $1(0.5 \%)$ & 0 & $1(1.3 \%)$ & \\
\hline Upper limb venous thrombosis & $1(0.5 \%)$ & $1(0.8)$ & 0 & \\
\hline Pocket hematoma & $1(0.5 \%)$ & $1(0.8)$ & 0 & \\
\hline Inappropriate shocks & $2(1 \%)$ & $2(1.6 \%)$ & 0 & \\
\hline Mortality & & & & 0.008 \\
\hline Related to device implantation & 0 & & & \\
\hline
\end{tabular}

* Data shown as absolute and relative frequency; ICD: implantable cardioverter-defibrillator; CRT-P/D: cardiac resynchronization therapy.

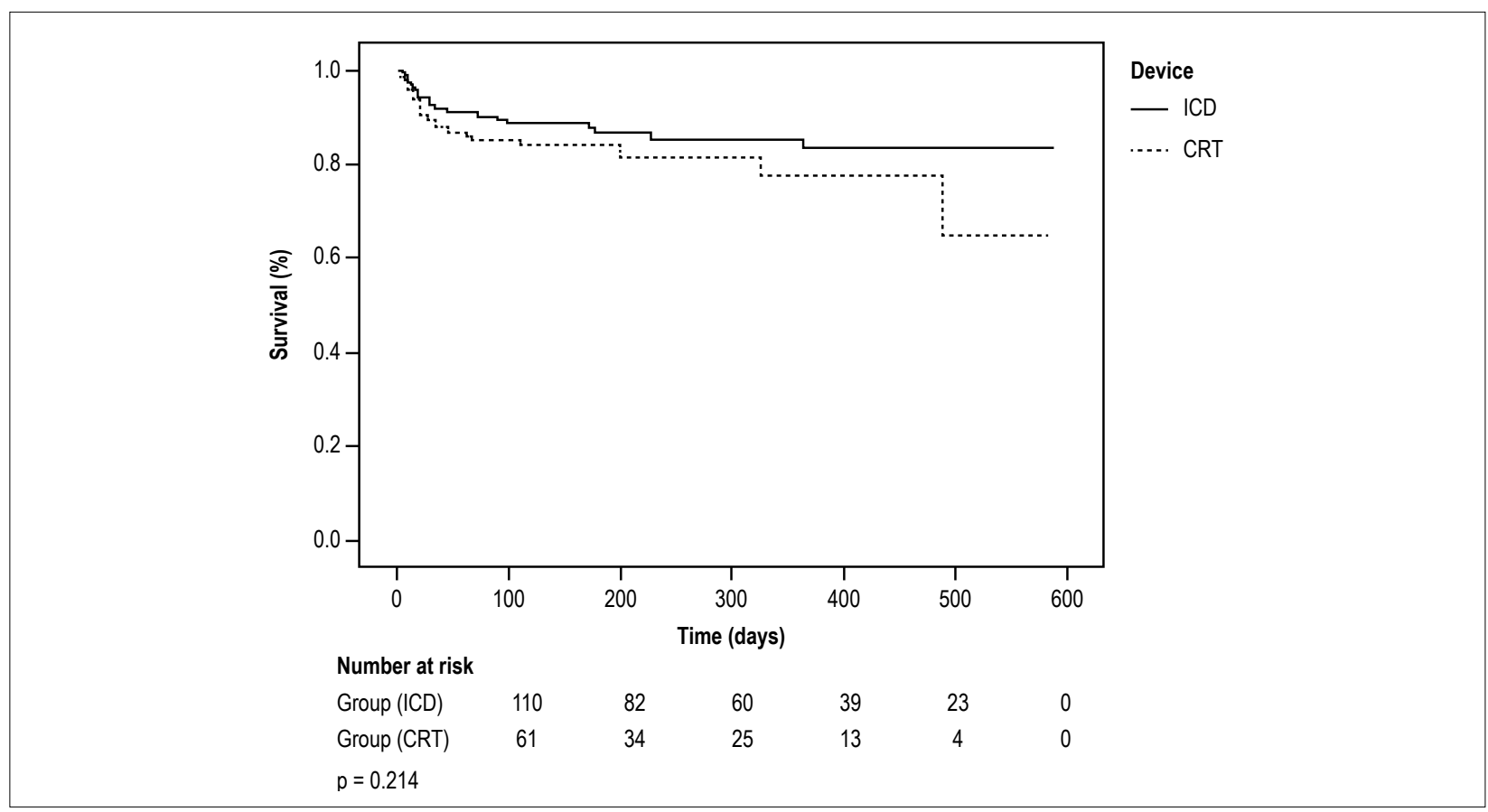

Figure 2 - Kaplan-Meier estimate of survival probability according to device-related unscheduled emergency visits. Note: $p=0.214$.

Van Rees et al., ${ }^{6}$ in a systematic review of 18 clinical trials involving ICD/CRT implantation, found a mortality rate of $2.7 \%$ after ICD implantation $(0.6 \%$ if considering only those without thoracotomy) and $0.7 \%$ after CRT. The incidence of lead dislodgement was $1.8 \%$ in the ICD group (without thoracotomy) and $5.9 \%$ in the CRT studies. Device pocket hematomas occurred in $2.4 \%$ of those with ICD and $2.2 \%$ with CRT; however, these percentages represented only cases that required surgical intervention. In our study, there was no difference between devices regarding the incidence of cable dislodgement, which is probably due to the low incidence of this complication in our sample. The incidence of CIED pocket hematoma found in our center was $0.8 \%$ in the ICD group and $0 \%$ in the CRT group.
When compared to other cohorts, we also found similar incidences of CIED-related complications. In a cohort of 1,929 patients, the incidence of surgical reintervention due to stimulation cable dislodgement, infection and mortality was, respectively, $4.4 \%, 1.5 \%$ and $3.2 \% .{ }^{9}$ Our cohort showed lower mortality and cable dislodgement rates, but $1 \%$ more incidence of bleeding. Among the patients with CRT, the incidence of cable dislodgement was $5 \%$, compared to $1.3 \%$ in our cohort.

A retrospective record of 30,984 Medicare users submitted to device implantation found an incidence of major complications (cable dislodgement, cardiac tamponade, hemothorax and pneumothorax) of $4.26 \%$, with no difference between CRT and ICD. ${ }^{12}$ In the same analysis, ICD implantation showed a higher incidence of mechanical 


\section{Original Article}

Table 3 - Outcomes of the study population. Unscheduled emergency visit unrelated to the device

\begin{tabular}{|c|c|c|c|c|}
\hline & Total $n=199$ & $I C D n=124$ & CRT-P/D n = 75 & $p$ value \\
\hline Unscheduled emergency visit unrelated to the device & $49(24.6 \%)$ & $35(28.2 \%)$ & $14(18.6 \%)$ & 0.17 \\
\hline Stroke & $1(0.5 \%)$ & - & $1(1.3 \%)$ & 0.79 \\
\hline Tiredness & $1(0.5 \%)$ & $1(0.8 \%)$ & - & 0.43 \\
\hline Headache/vertigo & $3(1.5 \%)$ & $3(2.4 \%)$ & - & 0.44 \\
\hline Glycemic disorders & $1(0.5 \%)$ & - & $1(1.3 \%)$ & 0.79 \\
\hline LUL pain - non-anginal & $2(1 \%)$ & $2(1.6 \%)$ & - & 0.70 \\
\hline Chest pain & $13(6.5 \%)$ & $9(7.2 \%)$ & $4(5.3 \%)$ & 0.81 \\
\hline Abdominal pain & $1(0.5 \%)$ & $1(0.8 \%)$ & - & 0.43 \\
\hline Heart failure & $18(9 \%)$ & $13(10.4 \%)$ & $5(6.6 \%)$ & 0.51 \\
\hline Acute lower limb ischemia & $2(1 \%)$ & $2(1.6 \%)$ & - & 0.70 \\
\hline Lower-limb myalgia & $1(0.5 \%)$ & - & $1(1.3 \%)$ & 0.79 \\
\hline Nausea/vomiting & $1(0.5 \%)$ & $1(0.8 \%)$ & - & 0.43 \\
\hline Pneumonia & $2(1 \%)$ & $1(0.8 \%)$ & $1(1.3 \%)$ & 0.71 \\
\hline Deep vein thrombosis & $1(0.5 \%)$ & $1(0.8 \%)$ & - & 0.43 \\
\hline Pulmonary thromboembolism & $1(0.5 \%)$ & - & $1(1.3 \%)$ & 0.79 \\
\hline Herpes zoster & $1(0.5 \%)$ & $1(0.8 \%)$ & - & 0.43 \\
\hline
\end{tabular}

${ }^{*}$ Data shown as absolute and relative frequency; ICD: implantable cardioverter-defibrillator; CRT-P/D: cardiac resynchronization therapy; LUL: left upper limb

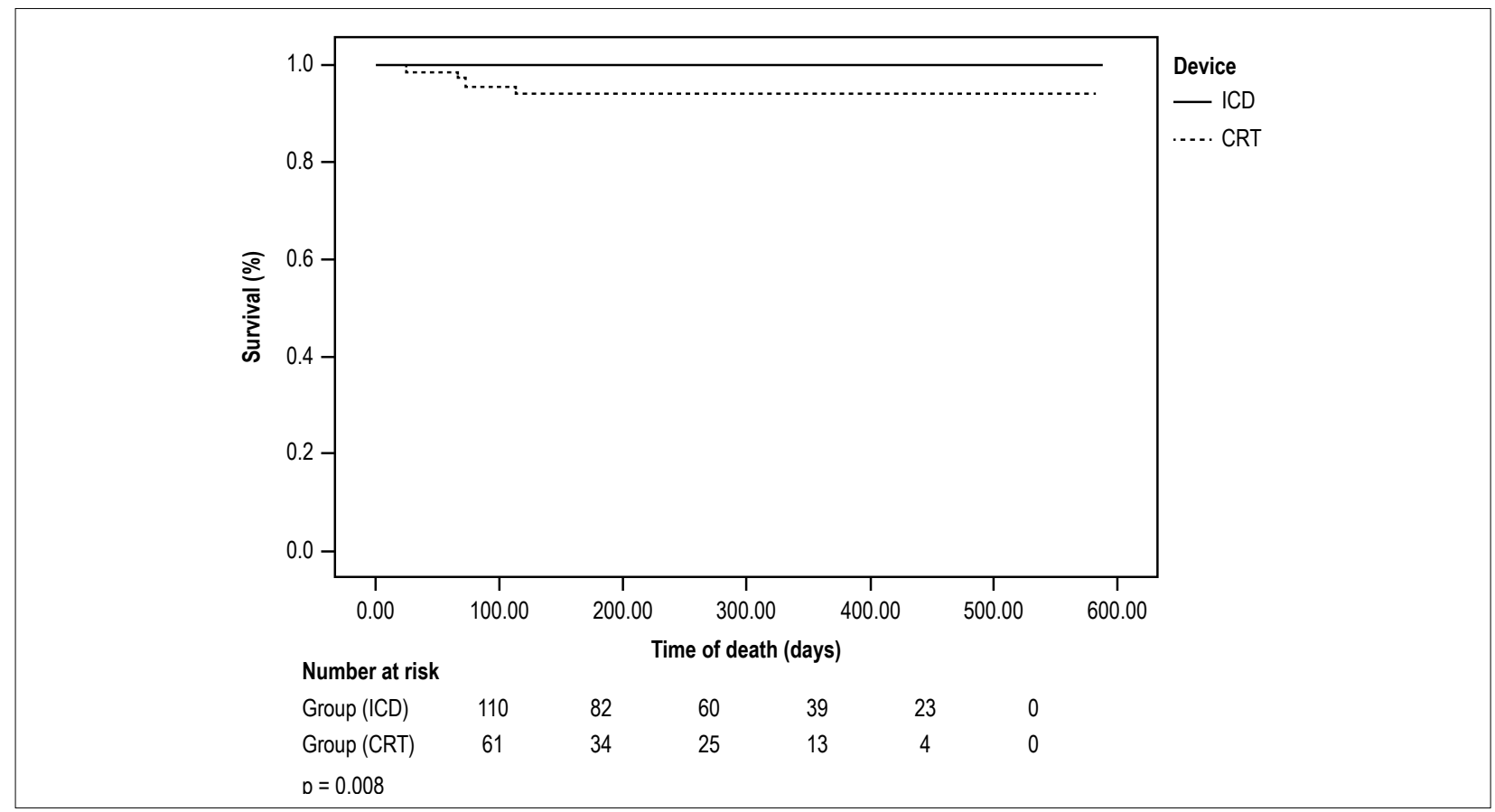

Figure 3 - Kaplan-Meier estimate of device-related survival probability. Note: $p=0.008$.

complications and infections, whereas CRT implantation showed a higher incidence of hematoma and hemorrhage findings that were opposite to those identified in our study.

In a prospective Dutch cohort of 1,517 patients, ${ }^{4}$ early complications were $9.2 \%$ and tended to a decrease after the first 6 months of implantation; the main ones, in order of frequency, were related to the cable in 5.54\% (3.34\% of dislodgement), device pocket $(4.75 \%$, excluding infection), hematoma $2.9 \%$, local trauma $(2.77 \%$ - with pneumothorax being $2.24 \%$ ) and pocket infection $(0.64 \%)$. In the late period, cable complications remained the same and pocket-related complications decreased, especially regarding the infection 
and local hematoma subcomponents. There was no evaluation of CRT and ICD implants, with the sample being restricted to the conventional pacemaker implant. Nevertheless, the rates of infection and cable dislodgement were higher than that recorded in the present study.

\section{Infections}

An analysis of the PEOPLE ${ }^{13}$ study (prospective cohort) evaluated 6,314 CIED implants at 44 centers. After 1 year, there were 633 deaths (10.1\%), 548 (8.9\%) non-infectious complications and 42 infections $(0.56 \%$ in patients submitted to the first CIED implantation). In the multivariate analysis, the factors related to a higher risk of infection were the occurrence of fever in the 24 hours prior to the implantation, use of a temporary pacemaker and the need for early reintervention. Our sample found an incidence of pacemaker pocket infection in $2.5 \%$ of cases. The higher incidence, albeit in accordance with the literature, may be due to the fact that we did not evaluate the de novo implants and did not collect data regarding the use of a temporary pacemaker prior to the procedure.

A Polish registry with 1,105 patients showed substantially lower infection rates when compared to the other recent studies: $0.1 \%$ at 2 months and $0.4 \%$ at the late follow-up of 2.4 years. ${ }^{14}$ Although the antimicrobial prophylaxis is a well-established outcome factor of protection, ${ }^{15,16}$ the registry differed from the others by the extended use of prophylaxis for a period of 5 days for surgical time $>1 \mathrm{~h}$ or immunosuppressive condition such as diabetes, chronic kidney disease, neoplasia or age $>75$ years. On the other hand, the percentages of device pocket hematoma were higher (6.1\%), associated with platelet antiaggregation, triple therapy or, mainly, anticoagulation (present in $56 \%$ of patients). If, on the one hand, there is evidence that the occurrence of hematoma increases by 15 -fold the risk of local infection, ${ }^{13}$ prospective studies on the duration of anticoagulation do not show worse outcomes with their maintenance in the peri-implantation period: on the contrary, they show a decrease of events. ${ }^{17,18}$ In a direct comparison between the two forms of stimulation, the ICD and CRT did not significantly differ, although absolute rates were higher in the ICD group.

In the present study, there was a difference between the groups regarding the proportions of ischemic etiology, which was higher in the ICD group, and in the functional class, characterized by higher class I proportions in the ICD group and class III in the CRT group. Among the non-device-related hospitalizations, the difference in functional class did not translate into a statistical discrepancy regarding the percentage of unscheduled visits, either in absolute numbers or the specific causal etiology (Table 3). Although there were no recorded deaths, the ICD group showed a higher proportion of important events, such as chest pain in the ischemic scenario, $\mathrm{HF}$ decompensation and acute limb ischemia. Compared to predictors of cardiac mortality models in resynchronization therapy, ${ }_{1}^{19,20}$ only $25 \%$ of the recorded deaths had an ejection fraction $<25 \%$ (specifically 28, 20, 58 and 29\%) and 75\% used loop diuretics at doses of $80 \mathrm{mg} /$ day or more. Right ventricular contractile dysfunction, an important factor associated with mortality, was not specifically analyzed in the present study. Inappropriate shocks, an important source of emergency consultations, were recorded in only 2 patients $(14.28 \%$ of device-related visits) - both with cardioverter defibrillators.

Compared with recent national data, ${ }^{21}$ the present study displayed a large difference regarding pocket hematoma rates, showing a much lower percentage in our cohort, but a higher percentage of unplanned device-related readmissions (7\% vs. $3.6 \%)$. It is important to emphasize that the current study did not account for the implantation of pacemakers without the ICD or CRT functions, situations that represented the majority of patients that were initially candidates for follow-up (Figure 1) and who, in fact, were included in a similar study, ${ }^{21}$ hindering the direct comparison between the findings. Furthermore, it should be remembered that the two assessed populations showed very different percentages of patients in functional class I and II (84.8\% vs. $31.5 \%$ in this study).

\section{Limitations}

Among the main limitations of the study is the small sample size when compared to the larger series, and the follow-up period duration, which may have been short for some outcomes and prevents the direct comparison with the larger cohorts in the literature; the analysis of the MIRACLE-ICD study subgroup,${ }^{19}$ for instance, suggests that left ventricular cable dislodgements becomes more frequent in the long term, so our follow-up may have underestimated the occurrence of this complication in the CRT group.

Also, we emphasize the fact that the data represent the practices and the results of a single cardiology center in the south of Brazil, with the limitations of unicentric studies on the extrapolation of results. Compared with recent local literature ${ }^{21}$ there are methodological differences regarding patient eligibility (mainly related to the type of device eligible for evaluation) and, consequently, considerable differences in baseline functional class and contractile function that limit the direct comparison between the studies regarding readmission predictors.

The difference between etiology and functional class between the groups is also a factor to be remembered. Although not statistically significant regarding the percentage of unscheduled visits to the emergency unit, regardless of whether or not it is associated with the procedure, the population's characteristics could lead to different results in the long-term follow-up, given the chronicity of the underlying diseases and their several forms of temporal evolution.

\section{Conclusion}

The results showed that patients submitted to CRT implantation, when compared to the ICD implantation cases, show a higher probability of mortality in the follow-up 


\section{Original Article}

period of less than 1 year. In contrast, the implant-related unscheduled hospital visit rate does not differ between the groups.

\section{Acknowledgment}

The authors thank the Research Unit of the Cardiology Institute / University Foundation of Cardiology for the technical support in the publication of this work.

\section{Author contributions}

Conception and design of the research, analysis and interpretation of the data and writing of the manuscript: Warpechowski Neto S, Ley LLG, Almeida ED, Saff MAL, Dutra LZ, Ley ALG, Sant`Anna RT, Lima GG, Kalil RAK, Leiria TLL; acquisition of data: Warpechowski Neto S, Ley LLG, Almeida ED, Saff MAL, Dutra LZ, Ley ALG, Lima GG, Kalil RAK; statistical analysis: Warpechowski Neto S, Ley LLG, Almeida ED, Dutra LZ, Ley ALG, Sant`Anna RT, Lima GG, Leiria TLL; obtaining funding: Kalil RAK, Leiria TLL; critical revision of the manuscript for intellectual content: Warpechowski Neto S, Ley LLG, Almeida ED, Saff MAL, Dutra LZ, Ley ALG, Sant`Anna RT, Lima GG, Kalil RAK, Leiria TLL.

\section{Potential Conflict of Interest}

No potential conflict of interest relevant to this article was reported.

\section{Sources of Funding}

This study was funded by FAPPIC (Fundo de Apoio à Pesquisa do Instituto de Cardiologia / Fundação Universitária de Cardiologia).

\section{Study Association}

This study is not associated with any thesis or dissertation work.

\section{Ethics approval and consent to participate}

This study was approved by the Ethics Committee of the Instituto de Cardiologia/Fundação Universitária de Cardiologia under the protocol number 5374/17. All the procedures in this study were in accordance with the 1975 Helsinki Declaration, updated in 2013. Informed consent was obtained from all participants included in the study.

\section{References}

1. Moss AJ, Zareba W, Hall WJ, Klein H, Wilber DJ, Cannom DS, et al; Multicenter Automatic Defibrillator Implantation Trial II Investigators. Prophylactic implantation of a defibrillator in patients with myocardial infarction and reduced ejection fraction. N Engl J Med. 2002;346(12):877-83.

2. Cleland JG, Daubert JC, Erdmann E, Freemantle N, Gras D, Kappenberger L, et al; Cardiac Resynchronization-Heart Failure (CARE-HF) Study Investigators. The effect of cardiac resynchronization on morbidity and mortality in heart failure. N Engl J Med. 2005;352(15):1539-49.

3. Sosa EA, de Paola AA, Martinelli M, Costa R, Pimenta J, Lorga AM, et al. [Recommendations of the Department of Arrhythmia and Clinical Electrophysiology (DAEC) of the Brazilian Cardiology Society on indications for permanent pacemaker implantation, choice of stimulation mode, and indications for automatic cardioverter-defibrillator implantation]. Arq Bras Cardiol. 1995;64(6):579-83.

4. UdoEO, ZuithoffNP, van Hemel NM, deCockCC, Hendriks T, Doevendans PA, et al. Incidence and predictors of short- and long-term complications in pacemaker therapy: the FOLLOWPACE study. Heart Rhythm. 2012;9(5):728-35.

5. Pakarinen S, Oikarinen L, Toivonen L. Short-term implantation-related complications of cardiac rhythm management device therapy: a retrospective single-centre 1-year survey. Europace. 2010;12(1):103-8.

6. van Rees JB, de Bie MK, Thijssen J, Borleffs CJ, Schalij MJ, van Erven L. Implantation-related complications of implantable cardioverter-defibrillators and cardiac resynchronization therapy devices: a systematic review of randomized clinical trials. J Am Coll Cardiol. 2011;58(10):995-1000.

7. Wilkoff BL, Auricchio A, Brugada J, Cowie M, Ellenbogen KA, Gillis AM, et al; Heart Rhythm Society; European Heart Rhythm Association; American College of Cardiology; American Heart Association; European Society of Cardiology; Heart Failure Association of ESC; Heart Failure Society of America. HRS/EHRA expert consensus on the monitoring of cardiovascular implantable electronic devices (CIEDs): description of techniques, indications, personnel, frequency and ethical considerations. Heart Rhythm. 2008;5(6):907-25.

8. Lee DS, Krahn AD, Healey JS, Birnie D, Crystal E, Dorian P JS, et al; Investigators of the Ontario ICD Database. Evaluation of early complications related to De Novo cardioverter defibrillator implantation insights from the Ontario ICD database. J Am Coll Cardiol. 2010;55(8):774-82.

9. Ghani A, Delnoy PP, Ramdat Misier AR, Smit JJ, Adiyaman A, Ottervanger $J P$, et al. Incidence of lead dislodgement, malfunction and perforation during the first year following device implantation. Neth Heart. J $2014 ; 22(6): 286-91$.

10. Ezzat VA, Lee V, Ahsan S, Chow AW, Segal O, Rowland E, et al. A systematic review of ICD complications in randomised controlled trials versus registries: is our 'real-world' data an underestimation? Open Heart. 2015;2(1):e000198.

11. Tobin K, Stewart J, Westveer D, Frumin H. Acute complications of permanent pacemaker implantation: their financial implication and relation to volume and operator experience. Am J Cardiol. 2000;85(6):774-6.

12. Reynolds MR, Cohen DJ, Kugelmass AD, Brown PP, Becker ER, Culler SD, et al. The frequency and incremental cost of major complications among medicare beneficiaries receiving implantable cardioverter-defibrillators. J Am Coll Cardiol. 2006;47(12):2493-7.

13. Klug D, Balde M, Pavin D, Hidden-Lucet F, Clementy J, Sadoul N, et al PEOPLE Study Group. Risk factors related to infections of implanted pacemakers and cardioverter-defibrillators: results of a large prospective study. Circulation. 2007;116(12):1349-55

14. Tajstra M, Gadula-Gacek E, Kurek A, Adamowicz-Czoch E, Olszowski D, Ostr $\square$ ga M, et al. Complications in recipients of cardioverter-defibrillator or cardiac resynchronization therapy: Insights from Silesian Center Defibrillator registry. Cardiol J. 2017;24(5):515-22.

15. Da Costa A, Kirkorian G, Cucherat M, Delahaye F, Chevalier P, Cerisier A, et al. Antibiotic prophylaxis for permanent pacemaker implantation: a metaanalysis. Circulation. 1998;97(18):1796-801. 
16. de Oliveira JC, Martinelli M, Nishioka SA, Varejão T, Uipe D, Pedrosa AA, et al. Efficacy of antibiotic prophylaxis before the implantation of pacemakers and cardioverter-defibrillators: results of a large, prospective, randomized, double-blinded, placebo-controlled trial. Circ Arrhythm Electrophysiol. 2009;2(1):29-34.

17. Birnie DH, Healey JS, Wells GA, Verma A, Tang AS, Krahn AD, et al; BRUISE CONTROL Investigators. Pacemaker or defibrillator surgery without interruption of anticoagulation. N Engl J Med. 2013;368(22):2084-93.

18. Cheng A, Nazarian S, Brinker JA, Tompkins C, Spragg DD, Leng CT, et al. Continuation of warfarin during pacemaker or implantable cardioverterdefibrillator implantation: a randomized clinical trial. Heart Rhythm. 2011;8(4):536-40.
19. Young JB, Abraham WT, Smith AL, Leon AR, Lieberman R, Wilkoff B, et al Multicenter InSync ICD Randomized Clinical Evaluation (MIRACLE ICD) Trial Investigators. Combined cardiac resynchronization and implantable cardioversion defibrillation in advanced chronic heart failure: the MIRACLE ICD Trial. JAMA. 2003;289(20):2685-94

20. Rocha EA, Pereira FT, Abreu JS, Lima JW, Monteiro Mde P, Rocha Neto AC, et al. Development and validation of predictive models of cardiac mortality and transplantation in resynchronization therapy. Arq Bras Cardiol. 2015;105(4):399-409.

21. Silva KR, Albertini CM, Crevelari ES, Carvalho El, Fiorelli AI, Martinelli M Filho, et al. Complications after surgical procedures in patients with cardiac implantable electronic devices: results of a prospective registry. Arq Bras Cardiol. 2016:107(3):245-56. 